\title{
Síndrome de desgaste profesional (burnout) en médicos de unidades de cuidados intensivos pediátricos en la Argentina
}

\author{
Physician's burnout in pediatric intensive care units \\ from Argentina
}

\begin{abstract}
Dra. María Eugenia Galván ${ }^{a}$ Dr. Juan C. Vassallo ${ }^{a}$ Dra. Susana P. Rodríguez ${ }^{a}$ Dra. Paula Otero ${ }^{c}$, Dra. María Mercedes Montonatia ${ }^{a}$ Dr. Gustavo Cardigni ${ }^{a}$, Dr. Daniel G. Buamscha ${ }^{b}$, Dr. Daniel Rufach ${ }^{b}$, Dra. Silvia Santos ${ }^{b}$, Dr. Rodolfo P. Moreno ${ }^{b}$, Dra. Mariam Sarlib y Miembros del Grupo de Investigación Clínica y Epidemiológica en Terapia Intensiva Pediátrica - Sociedad Argentina de Pediatría
\end{abstract}

\section{RESUMEN}

Introducción. En la actualidad hay escasez de médicos en unidades de cuidados intensivos pediátricos (UCIP). La causa de este déficit es multifactorial, pero el "fenómeno de burnout" o agotamiento profesional se ha descripto como relevante.

Objetivo. Analizar la situación del recurso hu-

a. Hospital Nacional de Pediatría "Prof. Dr. Juan P. Garrahan". Ciudad Autónoma de Buenos Aires, Argentina.

b. Comité Nacional de Emergencias y Cuidados Críticos. Sociedad Argentina de Pediatría.

c. Grupo de Trabajo de Informática Médica. Sociedad Argentina de Pediatría.

Correspondencia:

Dra. María E. Galván: eugegalvan31@gmail. com

Financiamiento:

Beca de investigación

"Fundación Garrahan" y Beca de iniciación Ramón Carrillo-Arturo Oñativia otorgada por la Comisión Nacional Salud Investiga del Ministerio de Salud; ambas posibilitaron la inclusión de becarios de investigación durante el proceso de desarrollo.

Conflicto de intereses: Ninguno que declarar.

Recibido: 17-4-2012 Aceptado: 4-6-2012
Métodos. Estudio observacional de corte transversal, mediante la administración de encuestas porvía electrónica; se usó el cuestionario Maslach Burnout Inventory. Se invitó a participar a médicos terapistas pediátricos que se desempeñaran en el sector público o privado de la Argentina durante al menos $24 \mathrm{~h}$ por semana.

Resultados. Fueron completadas 162 encuestas (tasa de respuesta 60\%). Presentaron alto riesgo de burnout en agotamiento emocional

\section{Grupo de Investigación Clínica y Epidemiológica en Terapia Intensiva Pediátrica - Sociedad Argentina de Pediatría:}

Dr. Gabriel Nievas (Nuevo Hospital Río Cuarto, Córdoba); Dra. Carmen Colman (Hospital Pediátrico "Dr. Avelino L. Castellán", Resistencia, Chaco); Dr. Tomás Iolster, Dr. Silvio Torres (Hospital Universitario Austral, Buenos Aires); Dr. Guillermo Fagalde (Hospital del Niño Jesús, Tucumán); Dr. Julio J. Trentadue (Fundación Favaloro, Hospital Universitario, Buenos Aires); Dr. Roberto Jabornisky (Hospital de Niños "Juan Pablo II", Corrientes); Dr. Mauro Nieto (Hospital Regional Río Gallegos, Santa Cruz); Dr. Leonardo Busso (Hospital El Cruce, Florencio Varela, Buenos Aires); Dra. Elida Vanella de Cueto (Hospital "Humberto J. Notti". Guaymallén, Mendoza); Dr. Luis Landry (Hospital Alemán, Buenos Aires). Dra. Graciela Rivello (Sanatorio Güemes, Buenos Aires); Dr. Javier Ponce (Hospital de San Juan, San Juan); Dra. Brenda Davidson, Dr. Facundo Jorro (Sanatorio de la Trinidad Mitre, Buenos Aires); Dra. Mónica Centeno, Dra. Laura Vega (Sanatorio San Cayetano, Buenos Aires); Dr. Roberto Tomassone (Policlínico Modelo de Cipolletti, Río Negro); Dr. Daniel Allende, Dra. Andrea Vélez (Policlínico Neuquén, Neuquén); Dra. Silvia Castellanos, Dra. Roxana Jaen (Hospital de Clínicas “Dr. José de San Martín", Buenos Aires); Dra María Althabe (Unidad de Cuidados Intensivos, UCI 35, Hospital Nacional de Pediatría "Prof. Dr. Juan P. Garrahan", Buenos Aires); Dr. Silvia Filippini (Unidad de Cuidados Intensivos, UCI 44. Hospital Nacional de Pediatría "Prof. Dr. Juan P. Garrahan", Buenos Aires); Dr. Eduardo Motto (Unidad de Cuidados Intensivos UCI, 45, Hospital Nacional de Pediatría "Prof. Dr. Juan P. Garrahan", Buenos Aires); Dra. Silvia Sáenz (Hospital de Niños de la Santísima Trinidad, Córdoba); Dr. Daniel Capra, Dr. Luis Faure (Hospital Nacional "Profesor Alejandro Posadas", Buenos Aires). 
40 terapistas $(25 \%)$, en realización personal $9(6 \%)$ y en despersonalización 31 (19\%). En forma combinada, 66 profesionales $(41 \%)$ tuvieron riesgo alto de burnout en alguna de las dimensiones; resultaron factores protectores e independientes de este riesgo estar certificado en la especialidad (OR 0,38 IC $95 \%$ 0,19-0,75) y trabajar en UCIP del sector público (OR 0,31 IC 95\% 0,15-0,65), mientras que hacer más de $36 \mathrm{~h} /$ semana de guardia aumentó el riesgo $\left(\mathrm{OR}_{\mathrm{A}} 1,94 \mathrm{IC} 95 \%\right.$ 1,1-3,85). El 31\% afirmó que no planeaba continuar con actividad en cuidados intensivos y $86 \%$ no pensaban continuar con guardias en los siguientes años. La mayoría de los encuestados comunicó que modificaciones en la práctica profesional (remuneración, cargos de planta, jubilación temprana, menor carga de guardia), podrían prolongar la expectativa de continuar con actividades en UCIP.

Conclusiones. Un porcentaje significativo de médicos que trabajan en las UCIP de la Argentina tienen alto riesgo de burnout y baja expectativa de continuar en la especialidad.

Palabras clave: Unidades de Cuidados Intensivos Pediátricos, agotamiento profesional, recursos humanos.

http:/ /dx.doi.org/10.5546/aap.2012.466

\section{INTRODUCCIÓN}

Los cuidados críticos se refieren a la atención médica de pacientes que presentan enfermedades graves, inestables o agudas. ${ }^{1}$ En nuestro país, desde 1997 el cuidado intensivo pediátrico se ha convertido en una subespecialidad pediátrica certificada. ${ }^{2}$ La presencia de médicos entrenados en cuidados críticos mejora la sobrevida de los pacientes y disminuye los costos en salud. Asimismo, deficiencias del recurso humano en estas unidades aumenta la morbimortalidad. ${ }^{3}$

Actualmente nos enfrentamos a una escasez de médicos capacitados para trabajar en UCIP. Desde las áreas de recursos humanos se ha señalado a la especialidad de terapia intensiva pediátrica como prioritaria en la necesidad de formación. En el ámbito internacional, la Comisión de Recursos Humanos de las Sociedades de Cuidados Críticos y Respiratorios (COMPACCS), ${ }^{4}$ detalla que la futura demanda de servicios de cuidados críticos en los Estados Unidos pronto excederá la capacidad del actual sistema prestacional. En el año 2007, la Sociedad Argentina de Terapia Intensiva advierte a la comunidad sobre la necesidad de contar con el doble de especialistas en terapia intensiva, lo cual significaba la incorporación de 700 profesionales, a fin de satisfacer la demanda. Sin embargo, en los últimos años no se cubren las vacantes para la formación de estos especialistas.

Las causas por las cuales los médicos abandonan la UCIP son múltiples; el "fenómeno de burnout" o síndrome de agotamiento profesional (burnout) ${ }^{5-8}$ se ha descripto como uno de los factores relevantes.
Se entiende como burnout, según Freudenberger, ${ }^{9}$ al "agotamiento de energía experimentado por los profesionales cuando se sienten sobrepasados por los problemas de los demás". Más recientemente, Maslach y Jackson ${ }^{10,11}$ lo definen como "una respuesta al estrés laboral crónico cuyos componentes son un sindrome de agotamiento emocional y/o físico, baja productividad laboral y una excesiva despersonalización". ${ }^{\prime}$

Existen informes que señalan que la mitad de los intensivistas pediátricos padecerían el burnout, ${ }^{12}$ si bien en Argentina no existen trabajos publicados. El relevamiento de UCIP por parte de la Sociedad Argentina de Pediatría en el 2005 lo refleja en parte. ${ }^{2}$

El objetivo de este trabajo fue analizar la situación del recurso humano en las UCIP de Argentina, mediante un relevamiento sistemático de las características y opiniones de médicos que se desempeñan en estas unidades. En forma específica, los objetivos fueron: a) describir las características de los médicos que trabajan en UCIP; b) identificar su nivel de capacitación, desarrollo profesional y condición laboral; c) medir el grado de burnout; d) explorar la opinión sobre la continuidad laboral, y e) analizar posibles asociaciones entre las variables analizadas con la presencia de burnout.

\section{POBLACIÓN Y MÉTODOS}

Se trata de un estudio observacional de corte transversal, mediante la administración de encuestas en forma sistemática, voluntaria y confidencial.

Fueron invitados a participar todos los médicos con actividad asistencial, presentes en UCIP de la Argentina del sector público o privado, que cumplieron con los criterios de inclusión: poseer una carga horaria asistencial en UCIP igual o superior a $24 \mathrm{~h}$ semanales y brindar el consentimiento para el registro y encuesta durante los meses asignados a la recolección de los datos (de mayo 2010 a junio 2011).

Para la identificación de los candidatos por encuestar se utilizaron diferentes estrategias: listados de sociedad científicas, listas de congresos de la especialidad, en redes o estudios multicéntricos previos y solicitud a jefes de servicio de las UCIP del país para referir los datos de su personal. Con estos listados se elaboró una base de datos en la que posteriormente se eliminaron los datos duplicados o erróneos.

El instrumento para la recolección prospectiva de datos fue una encuesta autoadministrada que 
constaba de 3 secciones: 1) introducción con fundamentos, objetivos, compromiso de confidencialidad y solicitud para completarla en forma voluntaria; 2) listado de preguntas para recolectar las variables del estudio y 3) cuestionario de burnout (Maslach Burnout Inventory - MBI). ${ }^{13-16}$

Se realizó una prueba piloto que consistió en el envío de la encuesta a 20 médicos terapistas, en quienes se evaluó su comprensión de ella y la confiabilidad del proceso técnico de envío y recepción. Las variables exploradas incluyeron características demográficas (sexo, edad, estado civil, hijos, lugar de residencia), aspectos relacionados con la capacitación en cuidado intensivo pediátrico y con el desarrollo profesional; además, se recolectaron características laborales y grado de satisfacción profesional.

Para evaluar el burnout se utilizó el cuestionario Maslach Burnout Inventory-Human Services Survey (MBI-SS), considerada la versión clásica del MBI (Maslach y Jackson, 1986) ${ }^{11}$ que consta de tres escalas que miden la frecuencia con que los profesionales perciben el agotamiento emocional (el profesional siente que no puede dar más de sí mismo, tanto en lo emocional como en lo afectivo), la despersonalización (desarrollo de actitudes o sentimientos negativos hacia los pacientes, visión deshumanizada del paciente) y baja realización personal (autoevaluación negativa, creencia

TABla 1. Características de los pediatras intensivistas que respondieron la encuesta

\begin{tabular}{|c|c|c|}
\hline \multicolumn{3}{|l|}{ Generales } \\
\hline \multicolumn{2}{|c|}{ Edad (promedio $\pm \mathrm{DE})$} & $42 \pm 7,4$ años \\
\hline \multicolumn{2}{|l|}{ Sexo masculino (n \%) } & $69(43 \%)$ \\
\hline \multicolumn{2}{|l|}{ Estado civil soltero } & $31(19 \%)$ \\
\hline \multicolumn{2}{|l|}{ Con hijos } & $115(71 \%)$ \\
\hline \multirow[t]{3}{*}{ Lugar de residencia } & CABA & $34(21 \%)$ \\
\hline & GBA & $40(25 \%)$ \\
\hline & Resto del país & $88(54 \%)$ \\
\hline
\end{tabular}

Capacitación y actividad científica

Programa de capacitación formal en UCIP

(residencia o beca post-básica) $124(77 \%)$

Certificación en UCIP $\quad 88(54 \%)$

Pertenencia a alguna sociedad científica $139(86 \%)$

Actividad de capacitación reciente (<5 años) 152 (94\%)

Actividad docente $75(46 \%)$

Actividad en investigación $77(48 \%)$

Publicación científica $\quad 40(25 \%)$

CABA: Ciudad de Buenos Aires; GBA: Gran Buenos Aires; DE: desvío estándar. de que nada puede cambiarse laboralmente ni que vale la pena intentarlo). Esta escala consta de 22 ítems divididos en 3 subescalas que exploran realización personal en 8 ítems, agotamiento emocional en 9 y despersonalización en 5; los sujetos valoran cada ítem con una escala de tipo Likert, en la que indican con qué frecuencia han experimentado en el último año la situación descrita en la pregunta. En la escala de agotamiento emocional una puntuación $\geq 27$, representa un alto grado de burnout; para la de despersonalización, una puntuación $>9$ significa grado alto; la escala de realización personal tiene una significación opuesta a las anteriores, de modo que, $<33$ puntos se considera alta realización personal y $>39$, baja.

El protocolo de investigación fue aprobado por el Comité de Ética y el Comité de Revisión Institucional del Hospital de Pediatría "Prof. Dr. Juan P. Garrahan".

Para el procesamiento de los datos se utilizaron códigos que aseguraron su confidencialidad. Se compararon las características de los profesionales según el grado de burnout; a los fines de este análisis se consideró como en alto riesgo de burnout a aquel profesional que poseía por lo menos este grado en alguna de las subescalas exploradas. Para el análisis bivariado se emplearon las pruebas de t, U Mann Whitney o exacta de Fisher, según correspondiera. Con el resultado final alto riesgo de burnout se construyó un modelo manual de regresión logística a los fines de evaluar factores independientes de riesgo o protección para burnout; la construcción del modelo se realizó con aquellos factores con una asociación igual o menor a $\mathrm{p}$ 0,10; se exploraron interacciones y colinealidad. La calibración del modelo se evaluó mediante la prueba de Hosmer-Lemeshow. El grado de significación se estableció en $<0,05$ y el paquete estadístico para el procesamiento de los datos fue Stata 10.0 para Windows.

\section{RESULTADOS}

Fueron completadas 162 encuestas $(60 \%$ de respuesta). Las principales características del grupo se presentan en la Tabla 1.

En promedio, los médicos habían estado trabajando en UCIP durante los últimos $11 \pm 7,5$ años, con valores de 1 a 32 años; en 94 casos (58\%) el profesional tenía menos de 10 años de antigüedad. El 40\% trabajaba en una sola institución, el $39 \%$ en dos y $21 \%$ de ellos en tres o más. La mayoría de los médicos (96\%) dijo trabajar en unidades que reunían condiciones de alta complejidad. La 
mitad desarrollaba su actividad en el sector público y privado, 61 (38\%) en el sector público y 19 $(12 \%)$ en el privado con exclusividad. Respecto de las características de los cargos que desempeñaban en la unidad con mayor carga horaria, 49 (30\%) eran jefes de la UCIP y $77(47 \%)$ tenían función de planta; sin embargo, 38 médicos (23\%) solo tenían actividad de guardia.

Un alto porcentaje (89\%) hacía guardias en forma regular y, en 66 casos, la carga semanal por guardia era superior a $36 \mathrm{~h}$. En relación a la relación contractual laboral, 119 (74\%) tenían relación de dependencia, mientras que el resto $(26 \%)$ trabajaba con contratos transitorios o por honorarios.
El 65\% (110) dijo estar en desacuerdo total-parcial con la remuneración y el 69\% (112) opinó que volverían a elegir nuevamente esta especialidad; el 31\% afirmó que no planeaba continuar con la actividad en UCIP y el $86 \%$ no consideraba continuar con guardias en los próximos años.

En el Gráfico 1 se muestra, según la antigüedad, expresada como categorías ordinales de años de trabajo en la UCIP, las opiniones sobre la continuidad en la especialidad y con la realización de guardias, observándose que aun aquellos médicos con pocos años de antigüedad no planean continuar ni hacer guardias en el futuro.

Adicionalmente, en el cuestionario se explo-

GRÁFICO 1. Opiniones de pediatras terapistas según antigüedad en la especialidad (\% de respuestas positivas)

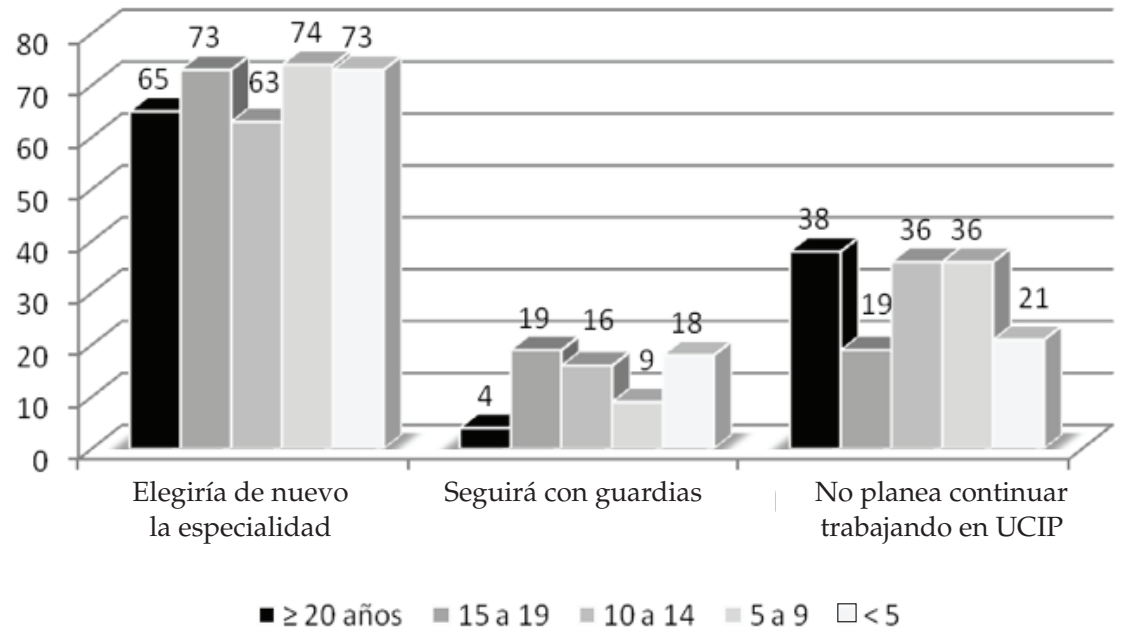

GRÁfICO 2. Número de profesionales según grado de riesgo en cada subescala del Maslach Burnout Index

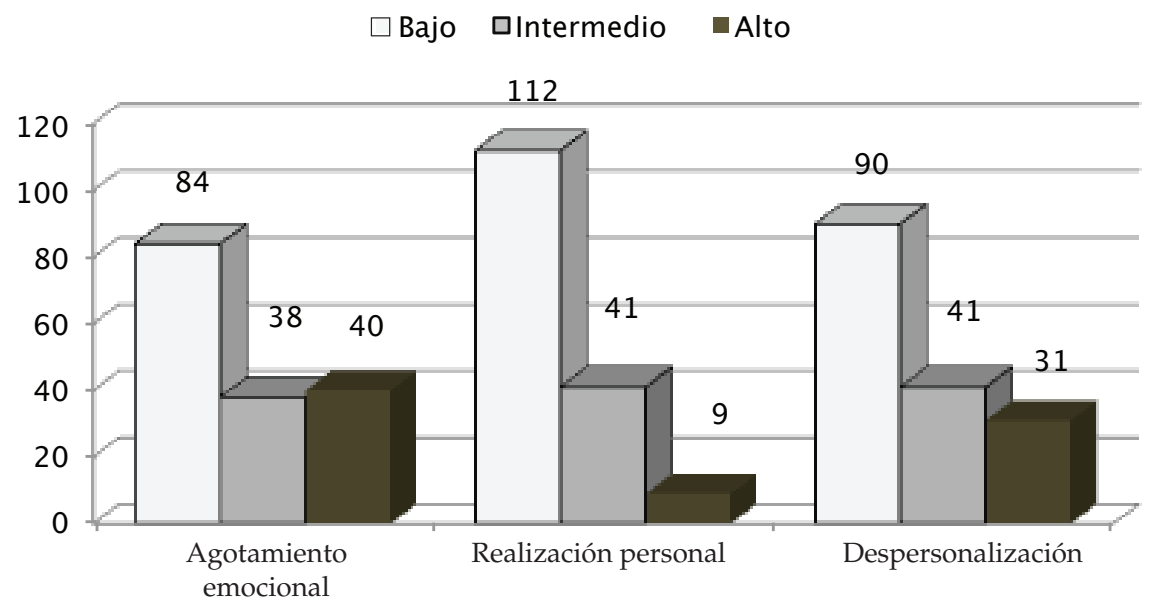


ró si existían condiciones que consideraban podrían fortalecer la continuidad en la actividad: 93\% (152) dijo que mejorar la remuneración, 70\% (114) señaló que anticipar la jubilación y 62\% (101) respondió que la disminución de la carga de horas de guardia y/o el aumento de la actividad de planta eran modificaciones que impactarían positivamente en la continuidad.

La medición de la escala de burnout mostró los siguientes resultados: para la subescala de agotamiento emocional el valor promedio fue de
$20 \pm 9,97$ puntos y el $25 \%$ (40 profesionales) tuvieron alto riesgo de burnout; para la subescala de realización personal, el valor fue de $30 \pm 7,15$ puntos y el 6\% (9 de ellos) tuvieron alto riesgo; finalmente, para la subescala de despersonalización el valor fue de 5,7 $\pm 4,8$ con el 19\% (31 terapistas) en alto riesgo. Al observar los datos en forma combinada, hallamos que 66 profesionales (41\%) tuvieron riesgo alto de burnout en alguna de las dimensiones o subescalas. En el Gráfico 2 se presenta el número de profesionales para ca-

TABLA 2. Comparación de las características de los profesionales según la presencia de alto riesgo de burnout

\begin{tabular}{|c|c|c|c|c|}
\hline & & $\begin{array}{l}\text { Con alto riesgo de burnout en alguna de las escalas } \\
\qquad 66(41 \%)\end{array}$ & $\begin{array}{l}\text { Sin alto riesgo } \\
\quad 96(59 \%)\end{array}$ & Valor $p$ \\
\hline \multicolumn{2}{|l|}{ Sexo masculino } & $31(47 \%)$ & $38(40 \%)$ & NS \\
\hline \multicolumn{2}{|l|}{ Edad X (DE) } & $41(7)$ & $43(7,5)$ & NS \\
\hline \multicolumn{2}{|l|}{ Soltero } & $19(29 \%)$ & $12(14 \%)$ & $0,01^{*}$ \\
\hline \multirow[t]{3}{*}{ Residencia en } & CABA & $16(24 \%)$ & $18(19 \%)$ & NS \\
\hline & GBA & $15(23 \%)$ & $25(26 \%)$ & \\
\hline & Resto del país & $35(53 \%)$ & $53(55 \%)$ & \\
\hline \multicolumn{2}{|l|}{ Tener hijos } & $41(62 \%)$ & $74(77 \%)$ & $0,03^{*}$ \\
\hline \multicolumn{2}{|c|}{ Años de trabajo en UCI X (DE) } & $9,48(6,9)$ & $12,18(7,8)$ & $0,02^{* *}$ \\
\hline
\end{tabular}

X: media; DE: desvío estándar; NS: no significativo; " test exacto de Fisher; ** test de U Mann-Whitney.

TABLA 3. Comparación de aspectos académicos según la presencia de alto riesgo de burnout

\begin{tabular}{|c|c|c|c|}
\hline & $\begin{array}{l}\text { Con alto riesgo de burnout en alguna de las escalas } \\
66(41 \%)\end{array}$ & $\begin{array}{c}\text { Sin alto riesgo } \\
96(59 \%)\end{array}$ & Valor $p$ \\
\hline Certificación & $27(41 \%)$ & $61(64 \%)$ & $0,004^{*}$ \\
\hline Capacitación formal & $54(82 \%)$ & $70(73 \%)$ & NS \\
\hline Actividad en capacitación reciente & $60(91 \%)$ & $92(96 \%)$ & NS \\
\hline Actividad docente reciente & $25(38 \%)$ & $50(52 \%)$ & NS \\
\hline Actividad de investigación & $28(42 \%)$ & $49(51 \%)$ & NS \\
\hline
\end{tabular}

* prueba exacta de Fisher.

TABLA 4. Comparación de aspectos laborales según la presencia de alto riesgo de burnout

\begin{tabular}{lccc}
\hline & Con alto riesgo de $\begin{array}{c}\text { burnout en alguna de las escalas } \\
\mathbf{6 6}(\mathbf{4 1} \%)\end{array}$ & $\begin{array}{c}\text { Sin alto riesgo } \\
\mathbf{9 6}(\mathbf{5 9} \%)\end{array}$ & Valor p \\
\hline Un lugar de trabajo & $22(33 \%)$ & $44(46 \%)$ & $\mathrm{NS}$ \\
Cargo de jefe & $18(27 \%)$ & $31(32 \%)$ & $\mathrm{NS}$ \\
Guardias & $62(94 \%)$ & $82(85 \%)$ & $\mathrm{NS}$ \\
Más de 36 horas semanales de guardia & $34(50 \%)$ & $32(33 \%)$ & $0,03^{*}$ \\
Solo actividad de guardia & $21(32 \%)$ & $17(18 \%)$ & 0,03 \\
Carga horaria mayor de 40 horas semanales & $15(23 \%)$ & $26(27 \%)$ & $\mathrm{NS}$ \\
UCIP alta complejidad & $61(92 \%)$ & $94(98 \%)$ & $\mathrm{NS}$ \\
Sector público & $16(24 \%)$ & $45(47 \%)$ & $0,003^{*}$ \\
Relación de dependencia & $44(67 \%)$ & $75(78 \%)$ & $\mathrm{NS}$ \\
\hline
\end{tabular}

NS: no significativo. * Prueba exacta de Fisher. 
da grupo de riesgo de burnout (bajo, intermedio y alto) en cada subescala.

El alto riesgo de burnout en la dimensión "realización personal" se mantuvo independiente del alto riesgo en las otras 2 escalas (ninguno de los 9 casos con alto riesgo tuvo agotamiento emocional ni despersonalización en ese grado de riesgo). A diferencia de esto, se observó asociación significativa entre agotamiento personal y despersonalización: el 35\% de los casos con alto riesgo en agotamiento emocional tuvo también alto riesgo en despersonalización (valor p 0,003).

En la Tabla 2 se comparan las características de los profesionales según el riesgo alto de burnout y se observa que aquellos con hijos y más antigüedad en la profesión tuvieron menor riesgo; por el contrario, ser soltero se asoció con mayor riesgo. En las Tablas 3 y 4 se observan los aspectos académicos y laborales de los médicos según la presencia de burnout; estar certificado y trabajar en el sector público disminuyó el riesgo, mientras que efectuar solo guardias y realizar más de $36 \mathrm{~h}$ de guardia semanal se asoció con mayor riesgo.

El análisis multivariado permitió estimar que fueron factores protectores e independientes de burnout estar certificado en la especialidad $\left(\mathrm{OR}_{\mathrm{A}}\right.$ 0,38 IC95\% 0,19-0,75) y trabajar en UCIP del sector público contra el privado $\left(\mathrm{OR}_{\mathrm{A}}\right.$ 0,31 IC 95\% $0,15-0,65)$ mientras que hacer más de 36 h de guardia semanal aumentó el riesgo $\left(\mathrm{OR}_{\mathrm{A}} 1,94 \mathrm{IC}\right.$ $95 \% 1,1-3,85)$.

\section{DISCUSIÓN}

Los resultados de este estudio muestran un elevado grado de burnout e insatisfacción laboral en los intensivistas pediátricos encuestados. En ella se encuentran reflejados pediatras de todas las jurisdicciones del país. En nuestro medio, otros informes han estimado una prevalencia similar. ${ }^{6}$

A diferencia del trabajo realizado por Pistelli y cols., en pediatras generales, que incluye personal en formación, ${ }^{7}$ los valores promedio de burnout observados en nuestro trabajo fueron menores para el agotamiento emocional $(20$ contra 30,6$)$ y realización personal (30 contra 35,7$)$, pero más elevados para la despersonalización (5,7 contra 0,5$)$; en dicho estudio, no se observan diferencias para los pediatras que realizan tareas de UCIP, si bien este subgrupo pudo haber estado menos representado; a su vez, la antigüedad en la profesión se asoció con mayor realización personal y menor despersonalización.
Nuestro estudio se enfoca exclusivamente en pediatras que son intensivistas, y si bien el mayor número de años de trabajo en la especialidad pareció proteger contra el riesgo de burnout global, esta asociación no se mantuvo en el análisis multivariado.

Los resultados de la escala de Maslach se pueden comparar mediante las medias aritméticas de las puntuaciones obtenidas por los sujetos para cada una de las tres subescalas (agotamiento emocional, despersonalización y realización personal) o situarlas en los intervalos correspondientes de burnout alto, medio o bajo preestablecidos para cada subescala. ${ }^{11}$ Las escalas no deben sumarse, ya que cada uno de los tres parámetros utiliza diferente sistema de puntuación e incluso de diferente signo, unas en positivo (más puntos, más burnout) y otra en negativo (más puntos, menos burnout). Otra forma de comparar resultados es contabilizar el porcentaje de los individuos estudiados que presentan una puntuación alta para una determinada subescala. A pesar de que no existe un grado global de burnout, hay autores que se refieren a un grado alto de burnout para aquellos sujetos con al menos una escala elevada. ${ }^{17,18}$ La importancia de la definición radica en la comparabilidad de los resultados con los de otros trabajos. Decidimos adoptar esta forma de análisis para caracterizar el riesgo en forma global, ya que el riesgo alto en cualquiera de las escalas significa una alerta para el profesional. De esta manera, el alto riesgo de burnout ocurrió en el $41 \%$ de los encuestados, lo cual implica un diagnóstico de situación alarmante. Recientemente se han publicado cifras de desgaste profesional cercanas al 38\% en oncólogos pediatras, donde nuevamente los más jóvenes presentaron la mayor frecuencia de burnout. ${ }^{19}$

Se han propuesto diferentes variables como factores predisponentes para el burnout: género, jornada laboral prolongada y pocas horas de sueño, entre otros. La situación de los profesionales más jóvenes parece ser un factor de riesgo en el que coinciden algunos estudios y resulta preocupante para el futuro de la especialidad. En un estudio realizado en Buenos Aires, la prevalencia de burnout entre los residentes en formación fue ya del $20 \%$, con alto componente de agotamiento emocional y despersonalización. ${ }^{20}$ En nuestro estudio, los médicos con menor antigüedad tuvieron mayor tendencia a presentar alto riesgo de burnout; adicionalmente, mostraron un alto porcentaje de insatisfacción y muy baja expectativa de continuar trabajando en UCIP, lo cual probablemente agrave la crisis de recurso humano en 
este sector. En nuestra población, tener más años ejerciendo la especialidad podría tener un efecto protector sobre la aparición de burnout.

La insatisfacción en la práctica médica se ha relacionado con diversos factores: el cuidado gerenciado, los aspectos legales, las mayores expectativas de los pacientes o las diferencias en la relación médico-paciente. Como señala Ceriani, en un reciente editorial, ${ }^{21}$ detrás de todos estos factores yace una preocupante situación de frustración y falta de motivación.

En nuestro estudio, un $80 \%$ de los profesionales no tiene expectativa de continuar trabajando en la especialidad en el futuro. Esta situación parece haber empeorado en los últimos años; un trabajo publicado en 1998 comunicó que el 55\% de los pediatras intensivistas encuestados en la Argentina estaban insatisfechos con su trabajo. ${ }^{8}$

Las condiciones laborales y el número de guardias se asocian con el riesgo de burnout. En nuestro estudio, trabajar en el ámbito privado o realizar más de $36 \mathrm{~h}$ de guardias por semana se asoció al desgaste profesional. Situaciones similares fueron informadas en intensivistas adultos en una reciente encuesta del Colegio Argentino de Terapia Intensiva. ${ }^{22}$ En ella describen que más de la mitad de los encuestados ejercían solo como médicos de guardia y realizaban un promedio de más de 48 h de guardia por semana.

La mayoría de los profesionales encuestados cree que implementar algunas medidas relacionadas con la práctica profesional, como jubilación temprana, guardias de $12 \mathrm{~h}$, adicional por terapia intensiva, entre otras, mejoraría la expectativa de continuar trabajando en las UCIP.

Las debilidades de nuestro trabajo están relacionadas con que un $40 \%$ de las encuestas no fueron contestadas y con el hecho de que no podemos descartar el temor a la identificación personal, lo cual podría influir sobre las respuestas.

\section{CONCLUSIONES}

Un $40 \%$ de los médicos que trabajan en las UCIP de la Argentina presenta un alto grado de burnout; pocos tienen expectativa de continuar trabajando en el área, y menos del 15\% considera continuar con la realización de guardias en los próximos años.

Estar certificado en la especialidad y trabajar en UCIP del sector público fueron factores protectores e independientes de burnout, en tanto que hacer más de 36 h de guardia semanal aumentó dicho riesgo.
Las modificaciones en la práctica profesional podrían prolongar la expectativa de los encuestados para desarrollar actividades asistenciales en la UCIP.

\section{Agradecimientos}

A la Dra. Virginia Sphers por su aporte en la revisión del protocolo de investigación.

\section{BIBLIOGRAFÍA}

1. Ewart QW, Marcus L, Gaba MM, Brandnen RH, et al. The critical care medicine crisis: a call for federal action a white paper from the critical care professional societies. Chest 2004;125(Issue 4,April).

2. Rufach D, Santos S, Vassallo J. Relevamiento de UCIP's en la República Argentina. IV Congreso Argentino de Emergencias y Cuidados Críticos Pediátricos. Buenos Aires, 2628 de mayo; 2005.

3. Joint commission on accreditation of Health Care Organization. Sentinel event alert preventing ventilator-related death and injuries. Washington, DC: Joint Commission; 2002. Pág.25.

4. Angus DC, Kelley MA, Schmitz RJ, White A, et al. Caring for the critically ill patient. Current and projected workforce requirements for care of the critically ill and patients with pulmonary disease: can we meet the requirements of an aging population? JAMA 2000;284:2762-70.

5. Cardigni G, Sphers V, Althabe M, et al. Burnout en terapia intensiva pediátrica en Argentina. $3^{\circ}$ Congreso Mundial Terapia Intensiva Pediátrica. Montreal Canadá;junio 2000.

6. Pistelli Y, Perochena J. Síndrome de desgaste profesional en médicos pediatras. Análisis bivariado y multivariado. Arch Argent Pediatr 2011;109(2):129-34.

7. Cardigni G, Althabe M, Vega L. El intensivista pediátrico ejerciendo la especialidad. Arch Argent Pediatr 1998; 96:363-8.

8. Maslach $\mathrm{C}$, Jackson $\mathrm{S}$. The measurement of experienced Burnout. J Occup Behavior 1981;2:99-113.

9. Freudenberger HJ. Staff burn-out. J Soc Issues 1974;30(1): 159-65.

10. Maslach C, Jackson SE. The Maslach Burn-out Inventory. Manual Research Edition. University of California. Palo Alto: Consulting Psychologist Press; 1986.

11. Maslach C, Schaufeli WB, Leiter MP. Job burnout. Ann Rev Psychol 2001;52:397-422.

12. Fields AL, Cuendon TT, Brasseux CO, Gets PR, et al. Physician burnout in pediatric critical intensive care medicine. Crit Care Med 1995;23:1425-9.

13. Gil-Monte PR. Factorial Validity of the Maslach Burn-out Inventory (MBIHSS) among Spanish professionals. Rev Saúde Pública 2005;39(1):1-8.

14. Gil-Monte PR, Peiró JM. A longitudinal study on Burnout syndromein nursing professionals. En: AvattoneF, Arnoldd eds. Burnout (Feelings work in Europe). Quaderni di Psicologia del Lavoro. Roma: Guerini Studio. 1997;5:407-14.

15. Gil-MontePR, Peiró JM. Validez factorial del Maslach Burnout Inventory en una muestra multiocupacional. Psicothema 1999;11(3):679-89.

16. Redondo Granado MJ. Errores frecuentes en la comparación de resultados de estudios sobre Burnout o desgaste profesional. An Pediatr (Barc) 2005;63(4):380-1.

17. Prieto Albino L, Robles Agüero E, Salazar Martínez LM, 
Daniel Vega E. Burnout en médicos de atención primaria de la provincia de Cáceres. Aten Primaria 2002;29:249-302.

18. Roth M, Morrone K, Moody K, Kim M, et al. Career burnout among pediatric oncologists. Pediatr Blood Cancer 2011;57(7):1168-73.

19. Catsicaris C, Eymman A, Cachiarelli N, Usandivaras I. La persona del médico residente y el síndrome de desgaste profesional (Burnout). Un modelo de prevención en la for- mación médica. Arch Argent Pediatr 2007;105(3):236-40.

20. Ceriani J. La insatisfacción en la práctica médica. Arch Argent Pediatr 2011;109 (6):474-5.

21. Colegio Argentino de Terapia Intensiva. Realidad Laboral del intensivista de la Argentina. $20^{\circ}$ Congreso Argentino de Terapia Intensiva. Mar del Plata 30 de septiembre al 3 de octubre de 2010. Disponible en: http:/ /www.cati.org. ar/files/medicos-intensivistas-un-recurso-critico-pdf.

Quizá haya enemigos de mis opiniones, pero yo mismo, si espero un rato, puedo ser también enemigo de mis opiniones. 